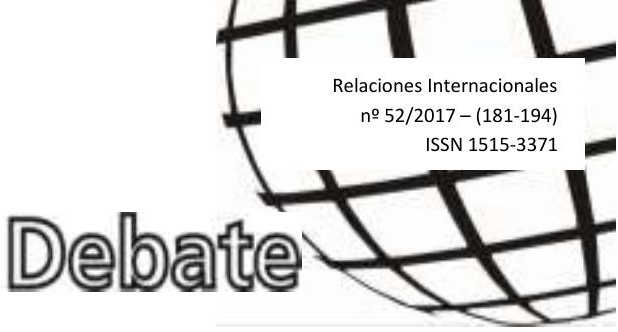

\title{
La EPI y las Relaciones Internacionales, ¿dónde está el Derecho? ${ }^{1}$ \\ IPE and International Relations: Where is the Law?
}

\author{
Juliana Peixoto Batista ${ }^{2}$
}

Resumen: Este trabajo apunta al debate teórico acerca de dos disciplinas fuertemente marcadas por la tradición anglosajona: las Relaciones Internacionales (RRII) y la Economía Política Internacional (EPI), sumando una disciplina que tiene tradición insoslayable en América Latina: el Derecho Internacional. Se construye como réplica a un trabajo sobre la EPI previamente publicado en esta revista (Tussie, 2015), donde se detallan las diferentes vertientes de la EPI, influencias, enfoques latinoamericanos, el contacto con otras disciplinas. Así, a lo largo de este artículo se identifican varios puntos de intersección y coincidencia entre las tres disciplinas, resaltando las visiones compartidas por las visiones críticas del Derecho y la EPI, donde se encuentran amplios espacios de colaboración conceptual, metodológica, desde la región latinoamericana en especial.

Abstract: This article aims at contributing to the discussion between two theoretical fields strongly influenced by the Anglo-Saxon's tradition - International Relations (IR) and International Political Economy (IPE) - by adding a third one, International Law (IL), as a significant field in Latin America. It is conceived as a response to a previous article published in this journal (Tussie, 2015). Tussie's paper detailed several IPE's strands, influences, linkages with other theoretical fields, and the Latin American contribution to IPE. With this in mind, several intersection areas among the three disciplines are identified in this paper, including similarities between critical legal studies and critical IPE as examples of those intersection spaces where we can find room for conceptual and methodological collaborative work.

Palabras Clave: Derecho Internacional, Relaciones Internacionales, Economía Política Internacional, América Latina

Keywords: International Law, International Relations, International Political Economy, Latin America

\footnotetext{
${ }^{1}$ Recibido: 14/03/2017. Aceptado: 09/06/2017

${ }^{2}$ Investigadora del Área de Relaciones Internacionales de FLACSO/Argentina. Es Doctora en Derecho y Magister en Relaciones Internacionales por la Universidad de Buenos Aires y Abogada por la Universidad Federal do Ceará (UFC, Brasil). Docente en la UBA, UNSAM y FLACSO en temas de integración regional, economía política internacional y su relación con el derecho internacional. jpeixoto@flacso.org.ar
} 


\section{Introducción}

Este trabajo se dedica a detallar los diversos puntos de intersección entre el Derecho y las Relaciones Internacionales (RRII), haciendo hincapié en la cercanía entre los enfoques más críticos del Derecho y la Economía Política Internacional (EPI). La idea de este articulo surge del trabajo de Diana Tussie, publicado en esta revista (Tussie, 2015). Dicho trabajo hace un resumen claro, amplio y enriquecedor del aporte de la EPI para el estudio de las RRII. Al leer el artículo, sin embargo, me percaté inmediatamente de la ausencia de una disciplina fundamental en dicha discusión, el Derecho. La EPI allí se detaIla de manera magistral, en sus diferentes vertientes, sus influencias, los enfoques latinoamericanos, el contacto con otras disciplinas, pero el Derecho está ausente. Luego de varias y acaloradas discusiones entre los y las investigadoras que nos sentimos parte constitutiva de este campo, he decidido sumar, al mencionado artículo, mi aporte de manera de ampliar la mirada e integrar los estudios y los ricos debates que se anclan en el Derecho. Busco en realidad seguir abriendo espacio al debate teórico acerca de dos disciplinas fuertemente marcadas por la tradición anglosajona: las RRII y la EPI, sumando una disciplina que tiene tradición insoslayable en América Latina: el Derecho Internacional (DI), disciplina instrumental desde la cual se insertaron a fines del siglo XIX las nuevas repúblicas latinoamericanas para hacer valer sus derechos como naciones soberanas, para detener las intervenciones militares que pretendían apropiarse de la renta aduanera, para el cobro de deuda soberana. Una disciplina que además hoy añade un instrumental interesante para ampliar la capacidad descriptiva y normativa (y en menor medida explicativa) del sistema internacional. Propongo entonces añadir que "la problemática de la EPI es fruto de una convergencia temática entre varias disciplinas: la Economía, la Historia, las Relaciones Internacionales, la Ciencia Política, la Sociología" (Tussie, 2015: 158) y el Derecho. En ese sentido, se puede decir que la EPI es un enfoque a través del cuál mirar al mundo, buscando conexiones invisibilizadas entre los enfoques tradicionales de las disciplinas de las que se nutre, proponiendo dejar al descubierto las relaciones de fuerzas, las desigualdades, las voces, los actores, las agendas que construyen el orden global, nacional, regional y/o internacional.

Se identifican a lo largo del trabajo varios puntos de intersección y coincidencia entre las RRII, el DI y la EPI. Buscamos ir más allá de los puntos de coincidencia más naturales entre el DI y las RRII que trajo la Escuela Institucionalista, para también abordar el surgimiento de un constitucionalismo internacional estrechamente vinculado con el liberalismo en las RRII, así como resaltar las visiones compartidas por el DI clásico y el realismo clásico - comúnmente entendidos como antagónicos absolutos en sus fundamentos -. Asimismo, como desde el DI y de las RRII se levantaron voces contrarias a esa visión liberal del mundo y desde donde la teoría crítica del Derecho y la EPI crítica encuentran amplios espacios de colaboración conceptual, metodológica, desde la región latinoamericana en especial.

Hay un punto crucial que habita esta discusión y que emerge más claramente de tanto en tanto a lo largo del trabajo: la disputa de poder subyacente entre las varias disciplinas, por ganar espacio en las (diversas pero relacionadas) comunidades epistémicas, resignificando conceptos y métodos. Esa dinámica se replica hacia adentro de las disciplinas mismas, en la disputa entre sus diferentes enfoques, en la construcción del sentido mismo de la disciplina en cuestión. Es intención de este trabajo asumir una posición con relación a este debate, expresando la riqueza de los enfoques interdisciplinarios 
que permiten abrir debates más que cerrarlos, y ampliar dimensiones de análisis que a su vez contribuyen a visibilizar agendas, intereses, dinámicas y actores en el escenario internacional.

En primer lugar, se hace un recorrido por los diversos puntos de encuentro entre las RRII y el DI, sobre todo por los debates que marcaron la segunda mitad del siglo XX y los primeros años del siglo $X X I^{3}$. Posteriormente, se retoman algunos puntos del artículo de Tussie (2015) para encontrar las diversas intersecciones entre la EPI y el Derecho. Al final, se esbozan algunas conclusiones.

\section{El Derecho Internacional y las Relaciones Internacionales}

El Derecho Internacional (DI) y las Relaciones Internacionales (RRII) comparten el contexto de estudio, el sistema internacional.

En muchas oportunidades, dan nombres diferentes a los mismos fenómenos y a menudo dialogan de manera más o menos tensa en la descripción del panorama internacional, sus causas y consecuencias. En palabras de Anne Marie Slaughter (1993), sería de gran ayuda para el avance del conocimiento si hicieran un esfuerzo por compartir vocabularios y marcos de análisis ya que miran el mismo mundo.

Así, las RRII surgieron como disciplina luego de lo que se diagnosticó como una incapacidad del Derecho Internacional de entender el sistema internacional y poder así mantener la paz y prevenir la guerra. Entre la Postguerra y los años 70 del siglo XX, se puede decir que teóricos como Kenan, Morghentau, Waltz sostenían desde el núcleo del realismo la hipótesis de que el Derecho como mucho cumplía un rol epifenomenal en la comunidad internacional (Slaughter, 1993). El realismo instaló la disciplina de las RRII que ganó rápidamente terreno con relación a la disciplina antes hegemónica, el Derecho Internacional (DI). De manera simplista, una de las máximas de la escuela realista afirma que es el poder, y no el Derecho, el que condiciona, restringe, modifica la conducta de los Estados. Si tomamos una definición clásica y amplia del Derecho (nacional o internacional) según la cual, éste sería una creación de la sociedad (nacional o internacional) y que, una vez creado, la modifica y la restringe (Celso Mello, 2001), se observa la diferencia estructural entre ambas perspectivas. A partir de ese desafío, lanzado a partir de la segunda mitad del siglo XX, el DI convive con la necesidad de demostrar su relevancia en el sistema internacional (Slaughter, 1993), o aun peor, demostrar que no es del todo irrelevante.

También es cierto que el realismo parte de una concepción de anarquía del sistema internacional para analizar la relación entre Estados soberanos, con el objetivo de buscar un orden que no sigue otra racionalidad que la del equilibrio de poder. En cambio, para el DI en general, hay una necesidad de coherencia del orden legal internacional, dado el supuesto de completitud del ordenamiento jurídico, lo que hace imperativa la necesidad de evitar la fragmentación y las contradicciones entre normas. Vale decir que el orden viene acompañado del concepto de seguridad jurídica cuyas dimensiones son previsibilidad y coherencia.

Sin embargo, esa perspectiva plantea solamente una de las facetas posibles de comparación entre las RRII y el DI. Para matizarla, hay que considerar al menos dos cuestiones más. En primer lugar, el realismo clásico y el DI clásico poseen también similitudes. De hecho, comparten una visión Estado céntrica del mundo y una visión simplista de la

\footnotetext{
${ }^{3}$ En ese sentido, ver también Peixoto Batista, Juliana, 2016.
} 
comunidad internacional, por lo que también fueron blanco de críticas por parte de nuevos enfoques tanto del Derecho como de las RRII. Dicho apartado tratará sobre ese debate. En segundo lugar, rescatamos otras relaciones posibles, como la cercanía entre las visiones (neo)estructuralistas de las RRII y los enfoques críticos del Derecho, que será objeto del apartado siguiente.

Con relación al primer debate, se puede decir que el Derecho Internacional clásico fue la disciplina articuladora del el sistema internacional desde la paz de Westfalia hasta la Primera Guerra Mundial. Un sistema normativo con una determinada ubicación geográfica (occidente), una inclinación ético-religiosa (cristiana), una inspiración económica (mercantilista-capitalista), fines políticos (imperialistas), además de un carácter negacionista de otras tradiciones jurídicas (Mello, 2001). En la visión de Tomuschat (1997), un derecho de convivencia, basado en la igualdad soberana de los Estados. O según Habermas (2008), un derecho de la coexistencia, entre Estados soberanos.

A partir de antecedentes como los del derecho humanitario internacional a fines del siglo XIX, el pacto Brian Kellog de principios del siglo XX y la incapacidad de DI de evitar una segunda guerra mundial, algunas de las características clásicas del DI fueron perdiendo fortaleza. El flagelo del Holocausto y la emergencia de EEUU como nueva potencia global dieron el puntapié para la reconfiguración de la disciplina. El liberalismo que inspiró el Nuevo Orden Mundial estableció entre otras cosas, la proyección del individuo como sujeto de Derecho Internacional por medio del Derecho Internacional de los derechos humanos, bajo el liderazgo de la Organización de las Naciones Unidas (ONU). Esa visión sostiene la restricción de las acciones de Estados soberanos para proteger derechos fundamentales de individuos, socavando así la visión clásica estadocéntrica y haciendo emerger un derecho de inspiración liberal neokantiana. Según Cottier (2002) el liberalismo es el ingrediente esencial del orden de la post Segunda Guerra Mundial e impuso fronteras y al mismo tiempo requerimientos positivos al Estado. En ese sentido, la Carta de la ONU -considerada por muchos como una especie de constitución de la comunidad internacional- establece como principios el Mantenimiento de la paz y la seguridad (Art. 1, 1 ); la Prohibición del uso de la fuerza (Art. 2, 4); el Arreglo pacífico de disputas (Art. 1, 1; art. 2, 3 y 33); los Derechos iguales y autodeterminación de los pueblos (Art. 1, 2); el Principio de cooperación, especialmente para fines económicos, culturales, sociales, humanitarios (Art. 1, 3); la Promoción del respeto a los derechos humanos y las libertades fundamentales, libre de discriminación (Art. 1, 3); la Igualdad soberana de todos los miembros (Art. 2,1).

Esa visión liberal del Derecho nutrió la emergencia del llamado constitucionalismo internacional, movimiento que luego conoció su auge en las últimas décadas del siglo XX, en medio del vertiginoso proceso de globalización y cima del pensamiento neoliberal. Dicha corriente teórica (Tomuschat, 1999; Habermas, 2000, 2008; Cottier y Hertig, 2003; De Wet, 2006, 2006å; Loughlin y Walker, 2008; Dunoff y Trachtman, 2009; Dobner y Loughlin, 2010), de marcada inspiración neokantiana, se basa en el término 'constitución' entendido como constitución moderna occidental. La novedad es que en esta oleada el constitucionalismo se expande más allá de las fronteras del Estado nación. Asimismo, describe los cambios ocurridos en el ámbito internacional como un proceso de constitucionalización del sistema internacional toda vez que dichos cambios están inspirados en valores constitucionales como ser los derechos humanos, la democracia, las reglas de gobernanza, la separación de los poderes, la creación de cortes internacionales, entre los principales. 
Además, el constitucionalismo internacional apunta a que a partir de la creación del nuevo orden, se observa una multiplicación de organizaciones internacionales que vinieron a regular los más variados aspectos de la vida internacional, disputando crecientemente protagonismo en la esfera internacional (Álvarez, 2006). Vale decir, que los cambios ocurridos se relacionan con las funciones del estado y su protagonismo en la Comunidad Internacional, y la pérdida relativa de su capacidad regulatoria y de protección de los individuos. Algunas de esas funciones y capacidades, según esta escuela estarían migrando a esferas internacionales (regionales o globales). Así, el constitucionalismo en el ámbito internacional, regional o global, puede ser descripto también como un proceso de des-estatización, resultado de la reducción de la capacidad regulatoria del Estado, y consiste en la transferencia de políticas tradicionalmente reguladas por el derecho doméstico hacia regímenes o estructuras de gobernanza internacional o supranacional, en una de migración de funciones constitucionales hacia el Derecho Internacional (Cottier y Hertig, 2003; Tsagourias, 2007; Dunoff y Trachtman, 2009). Según Giovanni Biagginni, "el derecho constitucional se torna cada vez más internacional, mientras que el Derecho Internacional se torna cada vez más constitucional" (citado en Cottier y Hertig, 2003: 269). En ese sentido, la función tradicional del Derecho Internacional estaría ahora suplementada por una función constitucional (Tomuschat, 1979).

Cottier (2002) afirma que los cambios en la capacidad regulatoria del Estado también son el resultado del comercio internacional, acelerado por el reciente y vertiginoso proceso de globalización. Para el autor, tanto los DD.HH. como el comercio internacional, a lo largo del siglo XX, desdibujaron las fronteras entre lo doméstico y lo internacional y establecieron estándares internacionales a ser alcanzados por todos los Estados. Acorde a ese planteo, Habermas $(2000 ; 2008)$ afirma que los DD.HH. constituirían el código moral de la normativa internacional básica que debe estructurar las reglas de convivencia entre los Estados. Así, el código legal en que se basa esa constelación posnacional es la Declaración Universal de los Derechos Humanos, cuya aplicación en los ámbitos nacional, regional, internacional, así como no gubernamental, es la principal y más legítima fuerza que está detrás de la creciente constitucionalización en el plano internacional.

La visión liberal del Derecho Internacional presenta, por lo tanto, muchos puntos de coincidencia con el liberalismo en las RRII, mayormente en lo que se refiere al protagonismo del individuo como unidad esencial de una comunidad, en detrimento del estado o grupos de estados. Asimismo, los cambios descriptos en el escenario internacional con relación a la emergencia de nuevos sujetos como las organizaciones internacionales, regionales, que disputan el protagonismo del Estado se corresponde en gran medida con una visión institucionalista del sistema internacional. Es decir, a primera vista, considerando la versión liberal del DI, los espacios de coincidencia y canales para la colaboración y retroalimentación más naturales serían el liberalismo y el institucionalismo en las RRII (Slaughter, 1993). De hecho, la llamada teoría de los regímenes -que en palabras del Anne-Marie Slaughter (idem) es Derecho Internacional con otro nombre- surgida a principios de los 80 es quizás uno de los mejores ejemplos de esa intersección al proponer un conjunto de conceptos y posibilidades metodológicas combinadas entre ambas disciplinas.

La teoría de los regímenes, posteriormente subsumida en la escuela Institucionalista de la RRII, fue quizás el ataque más frontal al núcleo de argumentos del realismo clásico respecto a su visión de las normas y las instituciones. Según dicha teoría, la multiplicación de los temas en la agenda internacional incrementó la demanda de regímenes internacionales, entendidos como complejos normativos más amplios que engloban no solo 
normas, sino también procedimientos, principios y procesos de tomas de decisiones (Krasner, 1982).

El término "régimen" se define como un conjunto de normas, principios, reglas y procedimientos de toma de decisiones, implícitos o explícitos, alrededor del cual las expectativas de los actores convergen en un determinado campo de las Relaciones Internacionales (Krasner, 1982). Para Keohane (1984: 97), los regímenes facilitan los acuerdos porque aumentan las posibilidades de anticipar los costos de la violación de las reglas, por proveer información fiable a los actores. Los regímenes se desarrollan en situaciones en que los actores tienen intereses comunes e intereses en conflicto acerca de temas múltiples que se solapan, en que las externalidades se manejan por medio de la negociación y el intercambio.

En la medida en que el mundo se complejiza, esa teoría sigue siendo revisada y actualizada y en los últimos años se ha mencionado la necesidad de analizar la interacción de regímenes, cada vez más necesaria, teniendo en cuenta el número cada vez mayor de regímenes internacionales y transnacionales, cada uno dotado de objetivos, instituciones y expertos (Raustiala y Victor, 2004; Alter y Meunier, 2009). Esta nueva situación ya no puede manejarse por medio de las relaciones interestatales tradicionales. Los problemas globales, como la protección del medio ambiente, la lucha contra el crimen organizado, y los objetivos como la prosperidad económica y la protección de los DD.HH. requieren interacción entre regímenes especializados a escala global (Twining, 2009).

Como se puede observar, la descripción del mundo en la visión de juristas e internacionalistas liberales es un campo fértil para la interdisciplinariedad. No obstante ello, esa cosmovisión ha recibido también muchas críticas y aquí también se observa un punto donde se abre espacio para el diálogo entre el DI y las RRII. De hecho, en contra del constitucionalismo internacional, se levantan internacionalistas y juristas, más afines a perspectivas críticas y definitivamente contrarios a la visión liberal del sistema internacional, según la cual la política y el derecho siguen un ideal basado en principios y valores racionales.

Así, desde el Derecho y desde la RRII, se ha criticado el constitucionalismo internacional afirmando que dicha corriente supone la existencia de valores universales (dichos constitucionales) que no existen. Según varios autores de la teoría crítica y/o más afines con visiones pluralistas del derecho, dicho enfoque deja poco margen a la pluralidad de actores y posiciones políticas (Stein, 2001; Rubenfeld, 2003; Koskenniemi, 2005, 2011; Kennedy, 2009; Rajkovic, 2010, entre otros), y trata de imponer el "pensamiento único" (Gill, 1998; Pureza, 2012). Martti Koskenniemi (2001, 2004, 2005, 2006a, 2011) considera que la diversidad es un hecho que debe ser reconocido, y que la corriente constitucionalista no es neutral con relación a diferentes compromisos políticos en disputa. Así, el autor afirma que no hay valores universales porque no hay, ni puede existir, una posición universal respecto de ellos y que los argumentos a favor de los valores universales expresan simplemente una intención hegemónica. El DI personifica valores particulares, específicos, de aquéllos que lograron imponerse hegemónicamente, pero que en ese camino muchas otras visiones válidas quedaron invisibilizadas. En ese sentido, Koskenniemi (ídem) al igual que Martha Finnemore (1996) rechazan la existencia de un camino progresivo hacia la armonización de las normas internacionales. Para ellos, las tensiones y contradicciones en el derecho dejan espacio para diferentes soluciones y arreglos y reflejan las tensiones y los conflictos entre los actores. Ello significa que no existe un ideal político y económico hacia donde converger. 
Por su parte, David Kennedy (2009) afirma que el debate sobre constitucionalismo internacional es en realidad un debate sobre gobernanza. Según el autor, en cada rincón del mundo se pueden ver los resultados de la globalización, pero muy poco se sabe sobre la forma en que operan esas muchas facetas del mundo globalizado (Kennedy, 2009: 67). Entender la forma en que el mundo globalizado funciona sería entonces la clave para empezar a ver el proyecto constitucionalista con más precaución. Hay que preguntarse dónde están las palancas, quiénes son las autoridades y cómo se relacionan entre sí en el mundo globalizado; si es todo simplemente caos y en qué medida es el trabajo de una mano invisible (ibíd.: p. 1). Sobre el debate específico del constitucionalismo y la llamada sociedad internacional, el autor cuestiona que la comunidad internacional esté realmente constituida. Pone en tela de juicio si se sabe lo suficiente sobre la estructura de los arreglos globales para estar tan confiados de que la estructura de constitucionalismo nacional es un buen modelo para aplicar en la esfera internacional. En esa misma dirección, Nikolas Rajkovic (2010) critica el optimismo de los académicos liberales con relación al rol del derecho en la gobernanza global. Dicho rol no sería un factor de restricción del poder de los Estados (rule of law), sino más bien una herramienta por medio de la cual los Estados más poderosos imponen las reglas (rule through law). El autor afirma que la gobernanza es una construcción social en donde las técnicas jurídicas son poderosas y centrales en la construcción de la "verdad social" y en consecuencia, de las conductas consideradas adecuadas.

Ello va en el mismo sentido de la crítica que hace Carl Schmitt (1986) al liberalismo. Schmitt critica el pensamiento normativista y la concepción de la política basada en el ideal de la discusión racional. Sostiene que la realización del Derecho depende de una decisión política vacía de contenido normativo. Desde una perspectiva ético-política, ello no implica la ausencia de valores y normas en la vida política sino la convicción de que éstos no pueden ser seleccionados por medio de un proceso de deliberación racional entre visiones alternativas del mundo. Valores y normas deben ser interpretados y decididos por quien detenta el poder (Negretto, 1995: 1).

Como se puede observar, las críticas apuntan a hacer visible la necesaria relación entre el poder y las normas en la esfera internacional, en afirmar el Derecho como un proceso político, como la cristalización en un dado momento histórico de una cierta correlación de fuerzas entre diferentes actores y grupos de intereses. En este punto se enlaza la articulación necesaria de la EPI con el Derecho.

\section{El enfoque de la economia política y los enfoques críticos del Derecho}

La visión del mundo compartida entre los teóricos críticos y los pluralistas desde el Derecho y las corrientes más estructuralistas desde las RRII, entre ellas la EPI, es otro campo de intercambio fértil entre ambas disciplinas.

Existen muchos cruces posibles entre dichos enfoques. Con relación a los enfoques críticos del Derecho, si bien son diversos y como tal surgieron en diferentes momentos históricos por diferentes razones en diferentes países, se puede decir que hay algunos hitos importantes. La Escuela Alemana de Frankfurt surgida en los años 20 del siglo XX es uno de ellos, así como los Critical Legal Studies, surgidos en EEUU en los años 70 del siglo $\mathrm{XX}$, los movimientos de la teoría crítica del derecho en países como Argentina, Brasil, Colombia, México - inspirados en el estructuralismo latinoamericano de los años 50 y 60 
- que fueron cruciales en los años 80 del siglo XX hasta el reciente pluralismo latinoamericano (también llamado constitucionalismo pluralista), con una fuerte impronta emancipatoria de pueblos originarios (Wolkmer, 2006). En líneas generales - y más allá de las batallas prácticas que han impulsado sea para reformar las instituciones del poder judicial, sea para promover cambios en la enseñanza del Derecho o en la formación de abogados que sean comprometidos socialmente - los enfoques críticos del Derecho (Koskenniemi, 2001, 2004 y 2006; Garavito, 2001; Gargarella, 2005 y 2010; Kennedy, 2009; Sousa Santos, 2009; Wolkmer, 2006) toman en consideración las variables políticas en el estudio del Derecho. En ese sentido, la aplicación del Derecho no responde a un ideal de racionalidad más allá de las relaciones de poder en una sociedad (nacional y/o internacional) y los diversos y muchas veces contrapuestos intereses que en ella conviven. EI DI estaría entonces compuesto por una multiplicidad de conjuntos normativos que presenta contradicciones, que a su vez no son salvables por medio de un proceso racional ya que son el reflejo de los intereses diversos de grupos sociales y políticos diversos. Asimismo, los enfoques críticos consideran que el DI generalmente responde a los intereses de los sujetos/Estados más favorecidos y poderosos, que logran casi siempre imponer sus demandas y protegerse de la acción de los sujetos/Estados menos favorecidos (Garavito, 2001; Gargarella, 2005; Rajovick, 2010; Sousa Santos, 2009). Por último, consideran que los actores sociales y políticos en el universo de acciones posibles que pueden adoptar, están restringidos por condicionamientos socio-económicos, políticos, culturales. Es decir, la estructura condiciona los agentes, por lo que los enfoques críticos suelen rechazar los conceptos liberales de autonomía y agencia plena.

De igual manera, la EPI desafía los conceptos de autonomía y agencia plena. No es de sorprenderse que la EPI en América Latina nace en el calor de la teoría de la dependencia, aunque para ese entonces, no tenía el nombre de EPI (Tussie, 2015). En ese entonces, bajo la insignia de desarrollo y los conceptos de centro-periferia - desarrollados en la obra central de Prebisch, Sunkel y Paz; de subimperialismo de Ruy Mauro Marini; de colonialismo interno de Pablo Gonzalez Casanova; del enfoque de la dependencia de Cardoso y Faletto-, se sentaron las bases de una sociología económica y una economía política (ídem: 157). En ese sentido, construye su primer corolario: la relación insoslayable entre economía y política. El origen de ese planteo es también el rechazo a los fundamentos del liberalismo (agencia plena, autonomía, estado mínimo) y su tendencia de autonomizar la esfera económica de la esfera política (Beigel, 2013).

Así, la problemática de la EPI se define por la influencia recíproca de la economía en la política (y viceversa) así como de lo interno en lo externo (y viceversa). Es decir, rechaza la separación entre "alta" y "baja" política. El Estado, aún el más poderoso, no tiene un poder omnímodo y abstracto a su entera disposición. La dinámica interna -en la cual se manifiestan diversos intereses en pugna, distintas modalidades de presión, diferentes grados de influencia de los actores sociales y políticos- fue eje de atención y fuente de explicación para comprender el comportamiento de los gobiernos cuya incidencia y participación en las cuestiones mundiales, ha ido creciendo en las últimas décadas. En breve, lo interno y lo externo no son vistos como compartimientos separados (ídem: 161). Aún más, no alcanza con fabricar un nuevo escenario transdisciplinario donde haya intereses económicos; además, es necesario sacar a la luz las palancas reales de poder. Implícitamente, la EPI hace una crítica al realismo como mirada unidimensional de la política. Las empresas son actores políticos no sólo porque pueden interferir en las prerrogativas del Estado, sino porque se relacionan de modo permanente con el Estado y sus 
diferentes estamentos. (ídem: 160). En dicho enfoque, algunos interrogantes son clave para los análisis: ¿quién se beneficia o puede beneficiarse con un determinado acuerdo o reglamentación? ¿quién gana y quién pierde con la ecuación de costos y beneficios?

Es interesante observar que si bien la EPI, como corriente de pensamiento, tiene fundamentos similares, sus resultados y motivaciones difieren bastante en diferentes momentos y contextos históricos. Así es que el surgimiento de la EPI con dicha denominación se da en los años 70 del siglo XX en EEUU, en el calor de las discusiones sobre la crisis del sistema de Bretton Woods, la crisis del petróleo de 1973 y la Guerra de Vietnam. Entre los conceptos más difundidos de ese enfoque en las RRII están la interdependencia, la transnacionalidad, la estabilidad hegemónica y los regímenes (Tussie, 2015). En cambio, el surgimiento de la EPI en el Reino Unido aporta a desdibujar las fronteras entre lo nacional y lo internacional al mismo tiempo que rechaza la preocupación por el concepto de estabilidad hegemónica, como algo inherente a EEUU y su preocupación por la declinación de su poder económico. Plantean así una EPI "no hegemónica". Sus supuestos estructuralistas y neomarxistas y su perspectiva sistémica la emparentan con los estudios de EPI en Latinoamérica (Tussie, 2015). A su vez, la vertiente latinoamericana, como ya mencionado, tiene sus raíces en el pensamiento estructuralista de la CEPAL y aporta conceptos importantes en los estudios de la EPI crítica como ser centro-periferia, autonomía, dependencia.

De igual manera, la teoría crítica del Derecho tiene peculiaridades derivadas de su contexto. Así es que la Escuela de Frankfurt surgió como una crítica a la modernidad y al pensamiento iluminista, rechazando la lógica instrumental y la racionalidad y rescatando el pensamiento de Marx ${ }^{4}$ y Freud, mientras que la escuela de Critical Legal Studies surge en los años 70 del siglo XX en EEUU como una crítica más dirigida al sistema de dominación maquillado de liberalismo occidental, a la ideología de los jueces en el dictado de sentencias (Kennedy, 2010), y defendía los derechos de minorías, la equidad de género, los derechos de los afrodescendientes. A su vez, en América Latina, la teoría critica del Derecho tuvo una marcada influencia del estructuralismo latinoamericano, y se nutrió de otras disciplinas como la pedagogía de Paulo Freire o la Teología de la Liberación y ha planteado desde movimientos emancipatorios de pueblos indígenas, como cuestiones del subdesarrollo o la capacitación de abogados militantes (Wolkmer, 2006).

Vale decir, tanto la teoría crítica del Derecho como el enfoque de la EPI crítica poseen una especificidad en América Latina que da lugar a un amplio espacio de intersección y mutua fertilización. Las preocupaciones sobre la autonomía, la dependencia, la emancipación de pueblos indígenas, la incidencia del poder económico en el Estado, las desigualdades del sistema internacional, el perfil de inserción llaman a profundizar en los análisis interdisciplinarios.

En ese sentido, hay muchos temas que cuentan con fuerte impulso en y/o desde América Latina. Para citar algunos ejemplos, la discusión sobre el régimen de protección de las inversiones y la deuda soberana; la relación entre las empresas y la violación de los Derechos Humanos; los avances en materia de consulta previa con relación a recursos naturales compartidos; los cambios en el régimen multilateral de comercio (GATT/OMC) para abarcar problemas del desarrollo y las asimetrías; los nuevos constitucionalismos pluralistas, entre muchos otras cuestiones actuales de interés. Todos esos temas parten

\footnotetext{
${ }^{4}$ Tambien es sabido que la Escuela de Frankfurt no es homogénea, y posee entre sus miembros Horkheimer, Marcuse y Habermas, pero resaltamos los fundamentos en su creación.
} 
de enfoques sistémicos críticos con relación al sistema internacional y se concretan mediante un esfuerzo de producción académica en las disciplinas relacionadas con la EPI: Relaciones Internacionales, Economía, Historia, Sociología, Derecho.

En el análisis de esos temas, es importante resaltar que una de las preguntas fundamentales de la EPI atañe fundamentalmente a la disciplina del Derecho: ¿quién se beneficia de un determinado acuerdo o reglamentación? En ese sentido, hay enfoques metodológicos que potencian la intersección entre ambos campos disciplinares. El estudio de cómo se construye determinada norma es uno de ellos. Los análisis de como las diferentes reglamentaciones responden a diferentes grupos de intereses en los niveles locales, regionales, nacionales es otro. Asimismo, al borrar las fronteras entre economía y política y entre lo doméstico y lo internacional, la EPI fomenta el estudio de sectores económicos transnacionalizados y aquí también el aporte del Derecho es crucial para entender los regímenes normativos, la influencia recíproca de las reglamentaciones públicas y privadas en la gestión de dichos sectores.

\section{Reflexiones finales}

Este trabajo surge de la necesidad de fomentar el dialogo entre dos disciplinas, el Derecho Internacional y las Relaciones Internacionales (RRII) las que, aunque se ocupen de lo mismo, caminan por senderos bifurcados en el mejor de los casos. En una visión menos positiva se puede decir que las RRII surgen como disciplina haciendo una poderosa crítica sobre la función del Derecho en el sistema internacional y como consecuencia de ello, parte de los años subsiguientes estuvieron dedicados, por parte de internacionalistas del Derecho, a demostrar la relevancia de las normas en la conformación y modificación del sistema internacional (Slaughter, 1993). Este artículo, que no pretende ser exhaustivo, se concreta como respuesta a un trabajo previo de Diana Tussie, publicado en esta misma revista en el año 2015 (Tussie, 2015), que aborda los fundamentos de la Economía Política Internacional (EPI). En dicho ejercicio, muy valioso para el estudio de la EPI, se omite una disciplina importante, el Derecho, que no sólo es una disciplina de mucha influencia en América Latina sino que desde sus enfoques más críticos, comparte con la EPI fundamentos, visión de mundo y son esenciales para un pensamiento latinoamericano sistémico y emancipatorio.

En ese sentido, hemos sugerido algunos campos de intersección posibles entre ambas disciplinas. En primer lugar, las coincidencias más claras, como ser el Derecho internacional de tinte más liberal como el constitucionalismo internacional por ejemplo, y el enfoque idealista en las RRII. En segundo lugar, el impulso de la escuela institucionalista, en unir los análisis jurídicos y de RRII en el estudio de los regímenes y de las organizaciones internacionales como actores cada vez más relevantes en el sistema internacional. De hecho, la EPI nace con esa denominación en los años 70 en EEUU, en el marco de la discusión coetánea sobre la necesidad de regímenes para ordenar el sistema internacional. En tercer lugar, las varias coincidencias entre los enfoques más críticos del Derecho y de la EPI, desde su marcada influencia estructuralista y neomarxista hasta su importancia para la concepción de América Latina como área de disputa disciplinar desde donde resignificar el sistema internacional. La región es, sin lugar a dudas, fundamental para las varias iniciativas que apuntan a disminuir las inequidades del sistema internacional a lo largo de la historia. 
En resumen, el campo de colaboración para trabajos interdisciplinarios es amplio. Por suerte, hoy por hoy, las posiciones extremas que sostienen la irrelevancia del Derecho en la conducta de los estados son minoritarias al mismo tiempo que en el Derecho, es creciente el interés por el estudio de las relaciones de poder subyacentes a las normas.

Koskenniemi (2001 y 2006) habla de la función hegemónica del Derecho. En ese sentido, cualquier disciplina puede ser herramienta de hegemonía en determinado contexto y momento histórico, como ha sucedido como la EPI en EEUU en los años 70. No es inherente a una u otra disciplina su carácter hegemónico, y sí su uso como instrumento de hegemonía. Por todo ello, es necesario rescatar el potencial de los estudios interdisciplinarios de Derecho y EPI como capaces de desafiar comunidades epistémicas instaladas y contribuir para el avance del conocimiento crítico y sistémico. El diálogo debe continuar.

\section{Bibliografía}

Abramovich, V. (2015). "Poderes regulatorios estatales en el pluralismo jurídico global”, Revista de Derecho Público, Año IV, n. 11, Infojus. Pp.3-31

Alter, K. y Meunier, S. (2009). "The Politics of International Regime Complexity", Perspectives on Politics, vo. 7, n. 01, pp.13-24.

Beigel, F. (2013). "Centros y periferias en la circulación internacional del conocimiento", Nueva Sociedad; No 245, mayo-junio.

Biaggini, C. (2000), "Die Idee der Verfassung - Neuausrichtung im Zeitalter der Globalisierung?"'", Zeitschrift für Schweizerisches Recht 119 (2000), 445 et seq. citado en Cottier, T. y Hertig, M. (2003). "The Prospects of 21st Century Constitutionalism", 7 Max Planck Yearbook of United Nations Law, 261-328

Bohoslavsky,J. P. (2010). Tratados de protección de las inversiones e implicaciones para la formulación de políticas públicas (especial referencia a los servicios de agua potable y saneamiento), Colección documento de Proyecto, CEPAL/GTZ/Ministerio Federal de Cooperación Económica y Desarrollo de Alemania, doc. LC/W.326.

Cottier, T. y Hertig, M. (2003). "The Prospects of 21st Century Constitutionalism", 7 Max Planck Yearbook of United Nations Law, 261-328.

De Wet, E. (2006). "The International Constitutional Order", International and Comparative Law Quarterly", vol. 55, January, pp. 51-76

(2006A). "The Emergence of International and Regional Value Systems as a Manifestation of the Emerging International Constitutional Order", 19 Leiden Journal of International Law. pp. 611-632.

Dobner, P. y Loughlin, M. (eds) (2010). The Twilight of Constitutionalism?, Oxford University Press.

Dunoff. J; y Trachtman, J. (eds.) (2009). Ruling the World? Constitutionalism, International Law, and Global Governance. Cambridge University Press.

Finnemore, M. (1996). National Interests in International Society, Cornell Studies in Political Economy Series, New York, Cornell University Press. 
Garavito, C. R. (coord.) (2011). El derecho en América Latina: un mapa para el pensamiento jurídico del siglo XXI, Buenos Aires: Siglo Veintiuno Editores. ISBN 978987-629-192-7

Gargarella, R. (2005). El derecho a la protesta. El primer derecho, Editorial Ad Hoc, Buenos Aires.

Gargarella, R. (2010). Legal foundations of Inequality. Constitutionalism in the Americas, 1776-1860, Cambridge University Press.

Gill, (1998). "European governance and new constitutionalism: Economic Monetary Union and alternatives to disciplinary neoliberalism in Europe", 3 New Political Economy. Pp. 5-15

Habermas, J. (2000). La constelación posnacional, Buenos Aires:Paidós.

(2008). El Derecho Internacional en la transición hacia un escenario posnacional. Europa: en defensa de una política exterior común (en colaboración con Jacques Derrida), Madrid: Katz editores y CCCB.

Kennedy, David. (2009). "The Mystery of Global Governance", en Dunoff. J; y Trachtman, J. (eds.). Ruling the World? Constitutionalism, International Law, and Global Governance. Cambridge University Press.

Kennedy, Duncan (2010). Izquierda y Derecho, Buenos Aires: Siglo XXI Editores.

Keohane, R. O. (1984). After Hegemony. Cooperation and Discord in the World of Political Economy, Princeton University Press

Koskenniemi, M. (2001) The Gentle Civilizer of Nations: The Rise and Fall of International Law 1870-1960, Cambridge University Press, 413 pp. (2004) "International Law and Hegemony: a Reconfiguration", 17 Cambridge Rev. of International Affairs 197, 202-203.

(2006). From Apology to Utopia: The Structure of International Legal Argument, Cambridge University Press.

Krasner, S (1982). "Structural Causes and Regimen Consequences", International Organization, 36. pp.185.

Loughlin, M. y Walker, N. (eds) (2008). The Paradox of Constitutionalism: Constituent Power and Constitutional Form, Oxford University Press. 392 pp.

Mello, C.D.A (2001). Curso de Direito Internacional Público, vol. I, 13a ed., Rio de Janeiro: Renovar.

Negretto, G. (1995). “¿Qué es el decisionismo? Reflexiones en torno a la doctrina política de Carl Schmitt", Revista Mexicana de Ciencias Políticas y Sociales, Vol 40, No 161

Nemiña, P. (2016). “Aportes de la Economía Política Internacional a la comprensión teórica de la relación entre el FMI y los países en desarrollo", CIVITAS, Porto Alegre, RS. 
Peixoto Batista, Juliana (2010). "Flexibilities for Developing Countries in the Doha Round as À La Carte Special and Differential Treatment: Retracing the Uruguay Steps", en Brazilian Journal of Latin American Studies, Cadernos PROLAM/USP, año 9, número 16, ene-jun, 2010, USP-LTr, San Pablo. pp. 164-191. ISSN 1676-6288. Disponible en http://www.usp.br/prolam/downloads/2010_1_9.pdf.

Peixoto Batista, Juliana (2016). - “Perspectivas jurídicas de las relaciones internacionales: el constitucionalismo internacional, la fragmentación del derecho y el pluralismo jurídico en debate", Revista TLAMELAUA, n. 39, Benemérita Universidad Autónoma de Puebla, México.

Programa Verdad y Justicia (2015). Responsabilidad empresarial en delitos de lesa humanidad. Represión a trabajadores durante el terrorismo de Estado. Tomos I y II. Ministerio de Justicia y Derechos Humanos de la Nación-CELS-FLACSO, Buenos Aires

Puerta, A.B. (2008). "El poder soberano y el devenir de la excepción en el pensamiento político moderno: Schmitt, Benjamin y Arendt ", Universidad de Antioquia, Colombia. Disponible en: http://bibliotecadigital.udea.edu.co/bitstream/10495/31/3/03_SchimittBenjam inArendt.pdf

Pureza, J. M (2012). "New Regionalism and Global Constitutionalism: Allies, Not Rivals", European society of International Law, Conference Paper Serires, n. 8/2012. 5a. Conferencia Bienal, Valencia-España, 13-15 de septiembre. Disponible en: http://papers.ssrn.com/sol3/papers.cfm?abstract_id=2193752\&rec=1\&srcabs= 2193746\&alg=1\&pos=1

Rajkovic, N. (2010). "Global Law and Governmentality: Reconceptualizing the Rule of Law and Rule Through Law", European Journal of International Relations, 18(1), 1952.

Raustiala, K. y Victor, D. (2004). "The Regime Complex for Plant Genetic Resources", International Organization, 58, 277.

Rubenfeld, J. (2003). "The Two World Orders", Wilson Quarterly, vol. 27

Schmitt, C. (1986). Political Romanticism, con traducción e introducción de Guy Oakes, Cambridge, Massachusetts, MIT Press.

Slaughter, A. M. (1993). "International Law and International Relations Theory: A Dual Agenda", American Journal of International Law, 87, 205.

Sousa Santos, B. (2009). Sociología jurídica crítica. Para un nuevo sentido común en el derecho, Editorial Trotta, Madrid.

Stein, E. (2001). "International Integration and Democracy: No Love at First Sight", 95 American Journal of International Law, pp.502-533.

Tomuschat, C. (1997). "International Law as the Constitution of Mankind", in United Nations (ed. ), International Law on the Eve of the Twenty-first Century. Views form the International Law Comission. 
Tsagourias, N. (ed.) (2007) Transnational Constitutionalism - International and European Perspectives, Cambridge University Press.

Tussie, D. (2015). “Relaciones Internacionales y Economía Política Internacional: Notas Para El Debate". Revista Relaciones Internacionales, v. 24, n. 48, julio. ISSN 2314-2766. Disponible en: http://revistas.unlp.edu.ar/RRIIIRI/article/view/2198

Twining, W. (2009). "Implications of Globalisation of Law as a Discipline", en Halpin y Roeben (eds.), Theorising the Global Legal Order, Oxford. Hart Publishing.

Walker, N. (2001) "The EU and the WTO: Constitutionalism in a New Key", en Burca, G y Scott, J. (eds.) The EU and the WTO: Legal and Constitutional Issues, Hart Publishing.

Wolkmer, A. C. (2006) Puralismo jurídico. Fundamentos de una nueva cultura del derecho. Editorial MAD, Sevilla. 